PROCEEDINGS OF THE

AMERICAN MATHEMATICAL SOCIETY

Volume 134, Number 2, Pages 371-378

S 0002-9939(05)08280-8

Article electronically published on August 25, 2005

\title{
RANDOM HOLOMORPHIC ITERATIONS AND DEGENERATE SUBDOMAINS OF THE UNIT DISK
}

\author{
LINDA KEEN AND NIKOLA LAKIC
}

(Communicated by Juha M. Heinonen)

\begin{abstract}
Given a random sequence of holomorphic maps $f_{1}, f_{2}, f_{3}, \ldots$ of the unit disk $\Delta$ to a subdomain $X$, we consider the compositions

$$
F_{n}=f_{1} \circ f_{2} \circ \ldots f_{n-1} \circ f_{n} .
$$

The sequence $\left\{F_{n}\right\}$ is called the iterated function system coming from the sequence $f_{1}, f_{2}, f_{3}, \ldots$ We prove that a sufficient condition on the domain $X$ for all limit functions of any $\left\{F_{n}\right\}$ to be constant is also necessary. We prove that the condition is a quasiconformal invariant. Finally, we address the question of uniqueness of limit functions.
\end{abstract}

\section{INTRODUCTION}

Suppose that we are given a random sequence of holomorphic self maps $f_{1}, f_{2}$, $f_{3}, \ldots$ of the unit disk $\Delta$. We consider the compositions

$$
F_{n}=f_{1} \circ f_{2} \circ \ldots f_{n-1} \circ f_{n} .
$$

The sequence $\left\{F_{n}\right\}$ is called the iterated function system coming from the sequence $f_{1}, f_{2}, f_{3}, \ldots$ By Montel's theorem (see for example [4]), the sequence $F_{n}$ is a normal family, and every convergent subsequence converges uniformly on compact subsets of $\Delta$ to a holomorphic function $F$. The limit functions $F$ are called accumulation points. Therefore every accumulation point is either an open self-map of $\Delta$ or a constant map. The constant accumulation points may be located either inside $\Delta$ or on its boundary.

We may look at the iterated function system as a dynamical system acting on $\Delta$. If $z$ is an arbitrary point of $\Delta$, its orbit under the iterated function system, $F_{n}(z)$, has $F(z)$ as an accumulation point. Hence, if the only limit functions are constants, the orbits of all points tend to periodic cycles. As we will see, we can find conditions so that whether this happens depends only on the subdomain $X \subset \Delta$ and not on the particular system chosen from $\mathcal{H o l}(\Delta, X)$.

If all maps in the iterated system are the same, the well-known Denjoy-Wolff Theorem determines all possible accumulation points.

Received by the editors March 8, 2004.

2000 Mathematics Subject Classification. Primary 32G15; Secondary 30C60, 30C70, 30C75.

The first author was partially supported by a PSC-CUNY Grant.

The second author was partially supported by NSF grant DMS 0200733 . 
The Denjoy-Wolff Theorem. Let $f$ be a holomorphic self-map of the unit disk $\Delta$ that is not a conformal automorphism. Then the iterates $f^{\circ n}$ of $f$ converge locally uniformly in $\Delta$ to a constant value $t$, where $|t| \leq 1$.

Therefore, whenever $f$ is not a biholomorphic isometry of $\Delta$, the system $f^{\circ n}$ has a constant accumulation point.

Many articles have studied possible generalizations of the Denjoy-Wolff Theorem to iterated function systems. One result of Lorentzen and Gill is

Theorem GL ([9], [12]). If an iterated function system is formed from functions in $\operatorname{Hol}(\Delta, X)$ where $X$ is relatively compact in $\Delta$, then the system $F_{n}$ converges locally uniformly in $\Delta$ to a unique constant. This constant is, of course, located in the relatively compact set $X$.

We say that a subdomain $X$ of $\Delta$ is degenerate if every iterated function system generated by a sequence of maps from $\Delta$ to $X$ has only constant accumulation points. Therefore, any relatively compact subdomain of $\Delta$ is degenerate, and moreover, each system has a constant limit.

A recent study of iterated function systems (see [3]) introduced new degenerate subdomains that are not relatively compact in $\Delta$. It also considered more general iterated function systems formed from maps in $\mathcal{H o l}(\Omega, X)$ where $X \subset \Omega$ are arbitrary plane domains such that $\Omega$ (and hence also $X$ ) admits $\Delta$ as a universal cover. We call such domains hyperbolic domains. In this context we say $X$ is degenerate in $\Omega$ if all the accumulation points of every iterated function system coming from $\mathcal{H o l}(\Omega, X)$ are constant. If $X$ is relatively compact in $\Omega$, we can apply Theorem GL to the universal covers of $\Omega$ and $X$ to show that $X$ is degenerate; again the interesting case is when $X$ is not relatively compact in $\Omega$.

The study cited above uses the Poincaré metrics on $\Delta, \Omega$ and $X$, denoted respectively by $\rho=\rho_{\Delta}, \rho_{\Omega}$ and $\rho_{X}$, as an important tool. The authors extended the classical Euclidean notion of a Bloch subdomain of the Euclidean plane to the hyperbolic setting. A subdomain $X$ of $\mathbb{C}$ is a Euclidean Bloch subdomain if, and only if, there is an upper bound on the radii of the disks lying in $X$. In the hyperbolic context we define

Definition 1.1. Let $R(X, \Omega)$ be the supremum of all radii (measured with respect to $\rho_{\Omega}$ ) of hyperbolic subdisks of $\Omega$ that are contained in $X$. A subdomain $X$ of $\Omega$ is called $a \rho$-Bloch subdomain of $\Omega$ if

$$
R(X, \Omega)<\infty .
$$

$R(X, \Omega)$ is called the $\rho$-Bloch radius of $X$ in $\Omega$.

In their paper, Beardon, Carne, Minda and $\mathrm{Ng}$ prove

Theorem BCMN. If $X$ is a $\rho$-Bloch subdomain of $\Omega$, then $X$ is degenerate in $\Omega$.

Example 1.2. Suppose that $\Omega$ is a plane domain obtained by removing at least two but only finitely many points from the whole complex plane. Suppose that $X$ is obtained by removing at least one but finitely many points from $\Omega$. Then any holomorphic map from $\Omega$ to $X$ has only removable singularities and therefore extends to a rational map of the whole complex sphere. It is easy to see that such a map cannot have an image in a strictly smaller subdomain, unless it is a constant map. Therefore, every holomorphic map from $\Omega$ to $X$ is a constant map and $X$ is degenerate in $\Omega$. Small punctured disks about each of the complementary points 
of $\Omega$ in $\mathbb{C}$ contain (non-schlicht) hyperbolic disks of arbitrarily large radii, so $X$ is a non- $\rho$-Bloch subdomain of $\Omega$. This example shows that the generalization of the converse of theorem BCMN does not hold: $X$ is non- $\rho$-Bloch in $\Omega$ but is degenerate. Furthermore, any iterated function system $F_{n}$ converges to the constant $f_{1}(z)$ and therefore the converse of the generalized version of Theorem GL does not hold either.

The situation is better, however, in the case when $\Omega$ is simply connected. In section 2 we prove

Theorem 1.3. Suppose that $X \subset \Delta$ is not a $\rho$-Bloch subdomain of $\Delta$. Then $X$ is not degenerate in $\Delta$.

Therefore, Theorem 1.3 together With Theorem BCMN implies

Corollary 1.1. $X$ is a $\rho$-Bloch subdomain of $\Delta$ if, and only if, $X$ is degenerate in $\Delta$.

In section 3 we show that $\rho$-Bloch subdomains are quasi-invariant with respect to the unit disk. More precisely we prove

Theorem 1.4. If $f$ is a quasiconformal self-homeomorphism of the unit disk $\Delta$, then $f$ maps every $\rho$-Bloch subdomain of $\Delta$ onto a $\rho$-Bloch subdomain of $\Delta$.

This theorem together with Corollary 1.1 implies

Corollary 1.2. If $f$ is a quasiconformal self-homeomorphism of the unit disk $\Delta$, then $f$ maps degenerate subdomains of $\Delta$ onto degenerate subdomains of $\Delta$.

In section 4 we turn our attention to the question of the uniqueness of limits of iterated function systems. We prove the converse of Theorem GL:

Theorem 1.5. Suppose that $X$ is any subdomain of the unit disk $\Delta$ that is not relatively compact in $\Delta$. Then there exists a sequence $f_{n}$ of holomorphic mappings from $\Delta$ to $X$ such that the iterated system $F_{n}=f_{1} \circ \ldots \circ f_{n}$ has more than one accumulation point.

Several recent articles are concerned with the study of iterated function systems and its applications, see for example, [3, 13, [14, [15. We would like to thank Fred Gardiner for numerous helpful discussions and remarks on an earlier version of this paper and Jonathan Brezin for his editorial advice.

\section{NON-CONSTANT ACCUMUlation POINTS}

In this section we prove Theorem 1.3. We begin by proving two preparatory lemmas. The first gives an estimate depending on the $\rho$-Bloch radius that relates the distances between relatively close points in the $\rho=\rho_{\Delta}$ and $\rho_{X}$ metrics. We normalize the density function for $\rho$ by $\rho(z)=\frac{1}{1-|z|^{2}}$.

Lemma 2.1. Let a be a point in a subdomain $X$ of $\Delta$. Let $C=R(X, \Delta, a)$ be the radius of the largest $\rho$-disk with center at a which is inside $X$. If $z$ is another point in $X$, with

then

$$
\rho(a, z)<1<C,
$$

$$
\rho_{X}(a, z) \leq(1+\epsilon) \rho(a, z)
$$


where

$$
\epsilon=\epsilon(C) \rightarrow 0 \text { as } C \rightarrow \infty .
$$

Proof. By applying the Möbius transformation

$$
A(z)=\frac{z-a}{1-\bar{a} z}
$$

we may assume that $a=0$. Suppose that $C=R(X, \Delta, 0)>1>\rho(0, z)$ for some point $z$ in $X$. Let $D$ be a disk in $\Delta$ with center at 0 and $\rho$-radius $C$. Then $D \subset X$, so that $\rho_{X} \leq \rho_{D}$. Therefore, an easy calculation shows that

$$
\rho_{X}(0, z) \leq \rho_{D}(0, z)=\int_{0}^{|z|} \frac{c}{c^{2}-t^{2}} d t
$$

where $c$ is the Euclidean radius of $D$. Obviously $c \rightarrow 1$ as $C \rightarrow \infty$. Therefore,

$$
\begin{aligned}
\rho_{X}(0, z) \leq & \int_{0}^{|z|} \frac{1}{1-t^{2}}\left(c+\frac{c\left(1-c^{2}\right)}{c^{2}-t^{2}}\right) d t \\
& \leq \int_{0}^{|z|} \frac{1}{1-t^{2}}\left(c+\frac{c\left(1-c^{2}\right)}{c^{2}-|z|^{2}}\right) d t \rightarrow \int_{0}^{|z|} \frac{1}{1-t^{2}} d t=\rho(0, z)
\end{aligned}
$$

as $c \rightarrow 1$, and the lemma follows.

The second preparatory lemma is about the contraction properties of Blaschke product maps of degree two.

Lemma 2.2. Let $c \neq 0$ be any point in $\Delta$ such that $\rho(0, c)<1$. If

$$
A_{a}(z)=\frac{z(z-a)}{1-\bar{a} z}
$$

then $A^{-1}(c)=\left\{z_{1}, z_{2}\right\}$ (that is, $A_{a}\left(z_{1}\right)=A_{a}\left(z_{2}\right)=c$ ) and

$$
\rho\left(0, z_{1}\right)=\rho\left(a, z_{2}\right) \rightarrow \rho(0, c) \text { as }|a| \rightarrow 1 \text {. }
$$

Proof. The two points $z_{1}$ and $z_{2}$ are the solutions of the equation $A_{a}(z)=c$ and thus roots of

$$
z^{2}-z(a-\bar{a} c)-c=0
$$

Therefore, we have

$$
z_{1} z_{2}=-c
$$

Since $A_{a}\left(z_{2}\right)=c$, by (2.2) we have

$$
\frac{z_{2}-a}{1-\bar{a} z_{2}}=\frac{c}{z_{2}}=-z_{1} .
$$

Thus,

$$
\rho\left(a, z_{2}\right)=\rho\left(0,-z_{1}\right)=\rho\left(0, z_{1}\right) .
$$

Solving equation (2.1), we obtain

$$
2 z_{1,2}=a-\bar{a} c \pm \sqrt{a^{2}+\bar{a}^{2} c^{2}+2 c\left(2-|a|^{2}\right)} .
$$

Hence,

$$
\begin{gathered}
2 a z_{1,2}=a^{2}-|a|^{2} c \pm \sqrt{a^{4}+|a|^{4} c^{2}+2 a^{2} c\left(2-|a|^{2}\right)} \\
\lim _{|a| \rightarrow 1}\left|2 z_{1,2}\right|=\lim _{|a| \rightarrow 1} \mid a^{2}-c \pm \sqrt{a^{4}+c^{2}+2 a^{2} c \mid} .
\end{gathered}
$$


Therefore we may order $z_{1}$ and $z_{2}$ so that

$$
\lim _{|a| \rightarrow 1}\left|2 z_{1}\right|=|2 c| \quad \text { and } \quad \lim _{|a| \rightarrow 1}\left|2 z_{2}\right|=\lim _{|a| \rightarrow 1}\left|2 a^{2}\right|=2 .
$$

Finally, $\lim _{|a| \rightarrow 1} \rho\left(0, z_{1}\right)=\rho(0, c)$, finishing the proof of this lemma.

Theorem 1.3. Suppose that $X \subset \Delta$ is not a $\rho$-Bloch subdomain of $\Delta$. Then $X$ is not degenerate in $\Delta$.

Proof. Let $X$ be any non- $\rho$-Bloch domain. We are going to construct an iterated function system from $\operatorname{Hol}(\Delta, X)$ with a nonconstant accumulation point. Pick any two distinct points $a_{0}$ and $w_{0}$ in $X$ such that $\rho_{X}\left(a_{0}, w_{0}\right)<1 / 2$. We will recursively find functions $f_{n}$ such that the iterated function system $F_{n}$ will have limit function $F$ that satisfies $F(0)=a_{0}$ and $F(\tilde{w})=w_{0}$ for some $\tilde{w}, \rho(0, \tilde{w})<1$.

First let $\pi_{1}$ be a universal covering map from $\Delta$ onto $X$ such that $\pi_{1}(0)=a_{0}$. Then there exists a point $c_{0} \in \Delta$, such that $\pi_{1}\left(c_{0}\right)=w_{0}$ and

$$
\rho\left(0, c_{0}\right)=\rho_{X}\left(a_{0}, w_{0}\right) .
$$

For any choice of $a_{1} \in X$, the Blaschke product $A_{a_{1}}$ produces two points $w_{1}$ and $\tilde{w}_{1}$ in $\Delta$ as the preimages of $c_{0}$ :

$$
A_{a_{1}}\left(\tilde{w}_{1}\right)=A_{a_{1}}\left(w_{1}\right)=c_{0} \text { and } \rho\left(0, \tilde{w}_{1}\right)=\rho\left(a_{1}, w_{1}\right) .
$$

Define $f_{1}=\pi_{1} \circ A_{a_{1}}$ so that

$$
\begin{gathered}
f_{1}(0)=f_{1}\left(a_{1}\right)=a_{0}, \\
f_{1}\left(w_{1}\right)=f_{1}\left(\tilde{w}_{1}\right)=w_{0} .
\end{gathered}
$$

We need to make sure that the point $w_{1}$ belongs to $X$. To do this we use the preparatory lemmas.

Let $\epsilon_{n} \rightarrow 0$ be a sequence such that $\Pi_{1}^{\infty}\left(1+\epsilon_{n}\right)^{2} \leq 2$. Since $X$ is non- $\rho$-Bloch, by Lemma 2.2. we can choose $a_{1} \in X$ so that $\left|a_{1}\right|$ is close enough to 1 so that

$$
\rho\left(0, \tilde{w}_{1}\right)=\rho\left(a_{1}, w_{1}\right)<\left(1+\epsilon_{1}\right) \rho\left(0, c_{0}\right)=\left(1+\epsilon_{1}\right) \rho_{X}\left(a_{0}, w_{0}\right) .
$$

Moreover, we may assume that $R\left(X, \Delta, a_{1}\right)$ is greater than 1 so that formula (2.7) implies that $w_{1} \in X$ and also that it is large enough so that by Lemma 2.1 and (2.7) we get

$$
\rho_{X}\left(a_{1}, w_{1}\right)<\left(1+\epsilon_{1}\right) \rho\left(a_{1}, w_{1}\right)<\left(1+\epsilon_{1}\right)^{2} \rho_{X}\left(a_{0}, w_{0}\right)<1 .
$$

Then, inductively, by our choice of $\epsilon_{n}$, there exist points $a_{n}, w_{n} \in X$ and $\tilde{w}_{n} \in \Delta$ such that

$$
\begin{gathered}
f_{n}(0)=f_{n}\left(a_{n}\right)=a_{n-1} \quad \text { and } \quad f_{n}\left(w_{n}\right)=f_{n}\left(\tilde{w}_{n}\right)=w_{n-1} \\
\rho\left(a_{n}, w_{n}\right)=\rho\left(0, \tilde{w}_{n}\right)<\left(1+\epsilon_{n}\right) \rho_{X}\left(a_{n-1}, w_{n-1}\right)
\end{gathered}
$$

and

$$
\rho_{X}\left(a_{n}, w_{n}\right)<\left(1+\epsilon_{n}\right) \rho\left(a_{n}, w_{n}\right)<\left(1+\epsilon_{n}\right)^{2} \rho_{X}\left(a_{n-1}, w_{n-1}\right) .
$$

Therefore

$$
\begin{gathered}
\rho\left(0, \tilde{w}_{n}\right)<\Pi_{1}^{n}\left(1+\epsilon_{i}\right) \rho\left(0, c_{0}\right)<1, \\
\rho_{X}\left(a_{n}, w_{n}\right)<\Pi_{1}^{n}\left(1+\epsilon_{i}\right)^{2} \rho\left(0, c_{0}\right)<1 .
\end{gathered}
$$

Now if $F_{n}=f_{1} \circ f_{2} \circ f_{3} \circ \ldots f_{n}$, the formulas 2.9 yield

$$
F_{n}(0)=a_{0} \quad \text { and } \quad F_{n}\left(\tilde{w}_{n}\right)=w_{0} .
$$


By Montel's theorem, $F_{n}$ is a normal family so that a subsequence of $F_{n}$ converges uniformly on compact subsets of $\Delta$ to a holomorphic limit function $F$. Therefore, equations (2.14) yield

$$
F(0)=a_{0} \quad \text { and } \quad F(\tilde{w})=w_{0},
$$

where $\tilde{w}$ is an accumulation point of the sequence $\tilde{w}_{n}$. Since, by equation (2.12), $\rho\left(0, \tilde{w}_{n}\right)<1$ for all $n$, the point $\tilde{w}$ belongs to $\Delta$. This implies that $F$ is a nonconstant function.

\section{DEGENERACY IN THE UNIT DISK IS A QUASICONFORMAL INVARIANT}

Since conformal homeomorphisms of the unit disk are hyperbolic isometries, conjugating an iterated function system by one preserves degeneracy. Here we show that the same is true for quasiconformal homeomorphisms.

Theorem 1.4. If $f$ is a quasiconformal self-homeomorphism of the unit disk $\Delta$, then $f$ maps every $\rho$-Bloch subdomain of $\Delta$ onto a $\rho$-Bloch subdomain of $\Delta$.

Proof. Suppose that $f$ is a $K$-quasiconformal map from the unit disk onto the unit disk and let $X$ be a non- $\rho$-Bloch subdomain of the unit disk. Then for every positive integer $n$ there exists a point $p_{n} \in X$ and a hyperbolic disk $D_{n} \subset X$ centered at $p_{n}$ with $\rho$-radius $n$. Suppose that $f(X)$ is $\rho$-Bloch. Then $R(f(X), \Delta)<\infty$. Let $F_{n}=h_{n} \circ f \circ g_{n}$, where

$$
g_{n}(z)=\frac{z+p_{n}}{1+\overline{p_{n}} z} \quad \text { and } \quad h_{n}(z)=\frac{z-f\left(p_{n}\right)}{1-\overline{f\left(p_{n}\right)} z} .
$$

Then $F_{n}$ is a $K$-quasiconformal map from the unit disk onto itself and $F_{n}(0)=0$.

Since $h_{n}$ is an isometry for $\rho$, there exists a point $q_{n}$ in $\Delta \backslash h_{n}(f(X))$ such that $\rho\left(q_{n}, 0\right) \leq R(f(X), \Delta)$. Since $g_{n}$ is an isometry for $\rho$, the hyperbolic disk $g_{n}^{-1}\left(D_{n}\right)$ with center at 0 and radius $n$ is contained in $g_{n}^{-1}(X)$. Therefore, $F_{n}^{-1}\left(q_{n}\right)$ must be outside the hyperbolic disk $g^{-1}\left(D_{n}\right)$. Thus, $\left|F_{n}^{-1}\left(q_{n}\right)\right| \rightarrow 1$ while $q_{n}$ stays bounded inside the unit disk. This contradicts the fact that the family of $K$-quasiconformal self-homeomorphisms of the unit disk fixing 0 is a normal family (see for example [2]).

Theorem (1.4) together with Corollary (1.1) obviously implies

Corollary 1.2. If $f$ is a quasiconformal self-homeomorphism of the unit disk $\Delta$, then $f$ maps degenerate subdomains of $\Delta$ onto degenerate subdomains of $\Delta$.

\section{Non-UNiqUENESS OF LIMIT POINTS}

In this section we study the question of the uniqueness of the limit points of an iterated function system in $\mathcal{H o l}(\Delta, X)$. A subdomain $X$ of $\Delta$ is degenerate if and only if it is a $\rho$-Bloch domain. We show that there are sequences in $\operatorname{Hol}(\Delta, X)$ that have more than one accumulation point. By Theorem GL, if the subdomain $X$ is relatively compact the limit points are unique, so we assume that $X$ is an arbitrary non-relatively compact subdomain of $\Delta$.

Theorem 1.5. Suppose that $X$ is any subdomain of the unit disk $\Delta$ that is not relatively compact in $\Delta$. Then there exists a sequence $f_{n}$ of holomorphic mappings 
from $\Delta$ to $X$ such that the iterated system $F_{n}=f_{1} \circ \ldots \circ f_{n}$ has more than one accumulation point.

Proof. Let $X$ be any subdomain of $\Delta$ that is not relatively compact. In our construction, all maps $f_{i}$ will be different universal covering maps from $\Delta$ onto $X$. We start with an arbitrary point $a$ in $X$. If we choose a point $a_{1} \neq a \in X$, we can find $f_{1}$ such that $f_{1}(a)=a_{1}$. Then $f_{1}$ is defined up to a (hyperbolic) rotation about $a$.

Let $f_{2}$ be a covering map from $\Delta$ onto $X$ such that $f_{2}(a)=a_{1}$ and such that there is an $a_{2} \in X$ with $f_{2}\left(a_{2}\right)=a$. There is such an $a_{2}$ because $X$ is not relatively compact in $\Delta$, so every hyperbolic circle with center at $a$ intersects $X$ and any covering map sending $a$ to $a_{1}$ is defined only up to rotation about $a$. Since covering maps are local isometries we may also assume $\rho\left(a_{2}, a\right)=\rho_{X}\left(a, a_{1}\right)$.

Continuing this process we obtain a sequence of covering maps $f_{n}$ and a sequence of points $a_{n}$ in $X$ such that

$$
f_{n}(a)=a_{n-1} \text { and } f_{n}\left(a_{n}\right)=a
$$

for all $n$. We study the even and odd subsequences of the iterated function system $F_{n}=f_{1} \circ \ldots \circ f_{n}$. The equalities (4.1) imply that the even subsequence $F_{2 n}$ satisfies $F_{2 n}(a)=a$, and the odd subsequence $F_{2 n+1}$ satisfies $F_{2 n+1}(a)=a_{1} \neq a$. Therefore these two subsequences have different accumulation points.

Theorems GL, BCMN, 1.3, 1.4 and 1.5immediately yield the following corollary.

Corollary 4.1. If $X$ is a subdomain of the unit disk $\Delta$, then:

(1) All accumulation points of any iterated function system of maps in $\mathcal{H o l}(\Delta, X)$ are constant functions if, and only if, $X$ is a $\rho$-Bloch-subdomain of $\Delta$.

(2) These accumulation points are unique if, and only if, $X$ is a relatively compact subdomain of $\Delta$.

(3) The properties in both (1) and (2) are preserved under quasiconformal selfhomeomorphisms of the unit disk.

\section{REFERENCES}

[1] L. V. Ahlfors, Complex Analysis, McGrawHill, (1953) MR0054016 (14:857a)

[2] L. V. Ahlfors, Lectures on Quasiconformal Mappings, Van Nostrand, (1966) MR 0200442 $(34: 336)$

[3] A. F. Beardon, T. K. Carne, D. Minda and T. W. Ng, Random iteration of analytic maps, Ergodic Th. Dyn. Systems, 24 (3), 2004, 659-675 MR2060992(2005b:37086)

[4] L. Carleson and T. W. Gamelin, Complex Dynamics, Springer-Verlag (1993). MR.1230383 (94h:30033)

[5] F. P. Gardiner, oral communication.

[6] _ Teichmüller Theory and Quadratic Differentials, Wiley-Interscience, 1987. MR0903027 (88m:32044)

[7] F. P. Gardiner and N. Lakic, Quasiconformal Teichmüller Theory, AMS Mathematical Surveys and Monographs, 76, 2000. MR1730906 (2001d:32016)

[8] F. P. Gardiner and N. Lakic, Comparing Poincaré densities, Annals of Math., 154, 2001, 245-267 MR:1865971 (2003c:30046)

[9] J. Gill, Compositions of analytic functions of the form $F_{n}(z)=F_{n-1}\left(f_{n}(z)\right), f_{n}(z) \rightarrow f(z)$, J. Comput. Appl. Math., 23 (2), 1988, 179-184 MR0959476 (89i:30028)

[10] L. Keen and N. Lakic, Forward Iterated Function Systems, in Complex Dynamics and Related Topics, Morningside Center of Mathematics, New Studies in Adv. Math IP Vol. $5,2003$.

[11] L. Keen and N. Lakic, An Introduction to Hyperbolic Geometry in the Small, In preparation

[12] L. Lorentzen, Compositions of contractions, J. Comput. Appl. Math., 32 1990, 169-178 MR.1091787 (92f:30035) 
[13] D. Mauldin, F. Przytycki and M. Urbanski, Rigidity of conformal iterated function systems, Compositio Math, 129 2001, 273-299 MR.1868356 (2003e:37062)

[14] V. Mayer, D. Mauldin and M. Urbanski, Rigidity of connected limit sets of iterated function systems, Mich. Math J., 49 2001, 451-458 MR.1872750 (2002j:37057)

[15] B. Solomyak and M. Urbanski, $L^{q}$ densities for measures associated with parabolic iterated function systems with overlaps, Indiana J. Math., 502001 MR.1889084 (2003i:28011)

[16] T. Sugawa and M. Vourinen, Some inequalities for the Poincaré metric of plane domains, preprint

Department of Mathematics, Lehman College and Graduate Center, CUny, Bronx, NEW YORK 10468

E-mail address: linda.keen@lehman.cuny.edu

Department of Mathematics, Lehman College and Graduate Center, CUny, Bronx, NEW YORK 10468

E-mail address: nikola.lakic@lehman.cuny.edu 